\title{
The pseudo core inverse of a companion matrix
}

\author{
Yuefeng Gao, Jianlong Chen ${ }^{\dagger}$ Pedro Patrício ${ }^{\ddagger}$ Dingguo Wang ${ }^{\S}$
}

\begin{abstract}
The notion of core inverse was introduced by Baksalary and Trenkler for a complex matrix of index 1. Recently, the notion of pseudo core inverse extended the notion of core inverse to an element of an arbitrary index in $*$-rings; meanwhile, it generalized the notion of core-EP inverse, introduced by Manjunatha Prasad and Mohana for complex matrices, to the case of $*$-ring. Many works have been done on classical generalized inverses of companion matrices and Toeplitz matrices. In this paper, we discuss the existence criteria and formulae of the pseudo core inverse of a companion matrix over a *-ring. In particular, a $\{1,3\}$-inverse of a Toeplitz matrix plays an important role in that process.
\end{abstract}

Keywords: pseudo core inverse; core inverse; core-EP inverse; companion matrix; Toeplitz matrix

AMS Subject Classifications: 15A09; 16W10; 16S50

\section{Introduction}

Throughout this paper, unless otherwise noted, $R$ is a $*$-ring, i.e., an associative ring with 1 and with an involution $*$. Let $R^{m \times n}$ denote the set of all $m \times n$ matrices over $R$ and let $A=\left(a_{i j}\right) \in R^{m \times n}$ with the involution $\left(a_{i j}\right) \rightarrow\left(a_{i j}\right)^{*}=\left(a_{j i}^{*}\right)$.

Let $a \in R$. $a$ is called (von Neumann) regular if $a$ has an inner inverse $a^{-}$, i.e., $a a^{-} a=a$. A $\{1,3\}$-inverse of $a$, denoted by $a^{(1,3)}$, is a solution to the equations $a x a=a$ and $(a x)^{*}=a x$; a $\{1,4\}$-inverse of $a$, denoted by $a^{(1,4)}$, is a solution to the equations $a x a=a$ and $(x a)^{*}=x a$. The Moore-Penrose inverse, Drazin inverse (resp. group inverse) and Drazin index of $a$ are denoted, as usual, by $a^{\dagger}, a^{D}$ (resp. $\left.a^{\#}\right)$ and $\operatorname{ind}(a)$ respectively.

Baksalary and Trenkler [1] introduced the notion of core inverse for a complex matrix, drawing lots of readers' attention. Then, Rakić et al. [13] extended this notion to an arbitrary

\footnotetext{
*E-mail: yfgao91@163.com Address: School of Mathematics, Southeast University, Nanjing 210096, China; CMAT-Centro de Matemática and Departamento de Matemática e Aplicações, Universidade do Minho, Braga 4710-057, Portugal

${ }^{\dagger}$ Corresponding author. E-mail: jlchen@seu.edu.cn Address: School of Mathematics, Southeast University, Nanjing 210096, China

${ }^{\ddagger}$ E-mail: pedro@math.uminho.pt Address: CMAT-Centro de Matemática and Departamento de Matemática e Aplicações, Universidade do Minho, Braga 4710-057, Portugal

${ }^{\S}$ E-mail: dgwang@qfnu.edu.cn Address: School of Mathematical Sciences, Qufu Normal University, Qufu, Shandong 273165, China
} 
*-ring. Later, Xu et al. [15] gave a characterization for the core (resp. dual core) invertible elements in $*$-rings in terms of three equations. The core inverse of $a$, denoted by $a^{\oplus}$, is the unique solution (if exists) to the equations

$$
x a^{2}=a, \quad a x^{2}=x, \quad(a x)^{*}=a x .
$$

We refer readers to [3] and [7] for a deep study of the core inverse. Since the core inverse was restricted to a square complex matrix of index 1, the notion of core-EP inverse [8] for an arbitrary square complex matrix was given by Manjunatha Prasad and Mohana as an extension of the core inverse. Then, the pseudo core inverse [4] extended the core-EP inverse from complex matrices to $*$-rings in terms of three equations. The pseudo core inverse of $a$, denoted by $a^{\circledR}$, is the unique solution (if exists) to the equations

$$
x a^{m+1}=a^{m} \text { for some positive integer } m, a x^{2}=x \text { and }(a x)^{*}=a x .
$$

The smallest positive integer $m$ satisfying the above equations is called the pseudo core index of $a$. If $a$ is pseudo core invertible, then it must be Drazin invertible, and the pseudo core index coincides with the Drazin index [4]. For convenience and consistency, we denote the pseudo core index of $a$ by $\operatorname{ind}(a)$. The pseudo core inverse is an outer inverse, i.e., $a^{\circledR} a a^{\circledR}=a^{\circledR}$. It is obvious that if the pseudo core index is equal to 1 , then the pseudo core inverse of $a$ is the core inverse of $a$.

These above definitions can be used, without modification, to characterize the existence of corresponding generalized inverses of matrices over rings.

Many works have been done on classical generalized inverses of companion matrices, for example, in [6] and [12], the authors considered the group inverse of a companion matrix; in [10] and [11], the Drazin inverse of a companion matrix was studied; in [9], the author gave necessary and sufficient conditions for a companion matrix to be Moore-Penrose invertible.

Inspired by the above papers, we are interested in considering the (pseudo) core inverse of a companion matrix. In Section 2, we focus on studying the core invertibility of a companion matrix over $R$. In Section 3, we consider the pseudo core invertibility of a companion matrix over $R$ and in this process, we find that a $\{1,3\}$-inverse of a lower triangular Toeplitz matrix over $R$ plays a key role. Therefore, in Sections 4 and 5 , a $\{1,3\}$-inverse of a lower triangular Toeplitz matrix is specially discussed.

\section{The core inverse of a companion matrix}

In this section, we consider the core invertibility of an $n \times n$ companion matrix $L_{n}=\left[\begin{array}{cc}0 & a \\ I_{n-1} & \beta\end{array}\right]$ over $R$, where $a=a_{0}, \beta=\left[a_{1}, a_{2}, \cdots, a_{n-1}\right]^{\mathrm{T}}$ and $I_{n-1}$ is an $(n-1) \times(n-1)$ identity matrix. Let us begin with a useful lemma.

Lemma 2.1. [12, Theorem 3] If $L_{n}=\left[\begin{array}{cc}0 & a \\ I_{n-1} & \beta\end{array}\right]$ with $\beta=\left[a_{1}, a_{2}, \cdots, a_{n-1}\right]^{\mathrm{T}}$ is a companion matrix over $R$. Then the following conditions are equivalent:

(1) $L_{n}^{\#}$ exists. 
(2) $a$ is regular with an inner inverse $a^{-}$and $h=a-\left(1-a a^{-}\right) a_{1}$ is invertible.

(3) $a$ is regular with an inner inverse $a^{-}$and $k=a-a_{1}\left(1-a^{-} a\right)$ is invertible.

In this case,

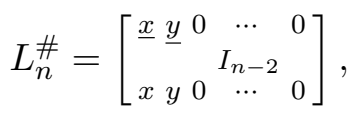

where $\underline{x}=\left[x_{1}, \cdots, x_{n-1}\right]^{\mathrm{T}}, \underline{y}=\left[y_{1}, \cdots, y_{n-1}\right]^{\mathrm{T}}$ and

$$
\begin{aligned}
& y_{i}=\delta_{i 1}+a_{i} y, \quad x_{i}=y_{i+1}+a_{i} x, \quad i=1, \cdots, n-1 . \\
& \left\{\begin{array}{l}
x=-\alpha_{1} a_{2} \alpha_{1}+\gamma_{1} \beta_{1}, y=\alpha_{1}, \quad n>2 \\
x=\alpha_{1}^{2}+\gamma_{1} \beta_{1}, y=\left[\begin{array}{ll}
\alpha_{1} & \gamma_{1}
\end{array}\right]\left[\begin{array}{ll}
\alpha_{1} & \gamma_{1} \\
\beta_{1} & \delta_{1}
\end{array}\right]\left[\begin{array}{c}
a_{1} \\
a
\end{array}\right], \quad n=2
\end{array}\right.
\end{aligned}
$$

$\alpha_{1}=-h^{-1}\left[1-a a^{-}\right], \quad \gamma_{1}=h^{-1} a a^{-}, \quad \beta_{1}=1+\left(a+a_{1}\right) h^{-1}\left[1-a a^{-}\right]$and $\delta_{1}=1-\left(a+a_{1}\right) h^{-1} a a^{-}$.

Theorem 2.2. If $L_{n}=\left[\begin{array}{cc}0 & a \\ I_{n-1} & \beta\end{array}\right]$ with $\beta=\left[a_{1}, a_{2}, \cdots, a_{n-1}\right]^{\mathrm{T}}$ is a companion matrix over $R$. Then the following conditions are equivalent:

(1) $L_{n}^{\oplus}$ exists.

(2) $a^{(1,3)}$ exists and $h=a-\left(1-a a^{(1,3)}\right) a_{1}$ is invertible.

(3) $a^{(1,3)}$ exists and $k=a-a_{1}\left(1-a^{(1,3)} a\right)$ is invertible.

In this case,

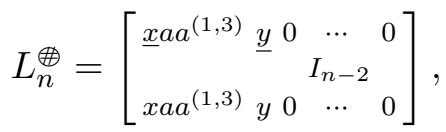

where $\underline{x}=\left[x_{1}, \cdots, x_{n-1}\right]^{\mathrm{T}}, \underline{y}=\left[y_{1}, \cdots, y_{n-1}\right]^{\mathrm{T}}$ and

$$
\begin{gathered}
y_{i}=\delta_{i 1}+a_{i} y, \quad x_{i}=y_{i+1}+a_{i} x, \quad i=1, \cdots, n-1 . \\
\left\{\begin{array}{l}
x=-\alpha_{1} a_{2} \alpha_{1}+\gamma_{1} \beta_{1}, y=\alpha_{1}, \quad n>2 \\
x=\alpha_{1}^{2}+\gamma_{1} \beta_{1}, y=\left[\begin{array}{ll}
\alpha_{1} & \gamma_{1}
\end{array}\right]\left[\begin{array}{ll}
\alpha_{1} & \gamma_{1} \\
\beta_{1} & \delta_{1}
\end{array}\right]\left[\begin{array}{c}
a_{1} \\
a
\end{array}\right], \quad n=2
\end{array}\right.
\end{gathered}
$$

$\alpha_{1}=-h^{-1}\left[1-a a^{(1,3)}\right], \quad \gamma_{1}=h^{-1} a a^{(1,3)}, \quad \beta_{1}=1+\left(a+a_{1}\right) h^{-1}\left[1-a a^{(1,3)}\right]$ and $\delta_{1}=1-\left(a+a_{1}\right) h^{-1} a a^{(1,3)}$.

Proof. Consider the factorization of $L$ :

$$
L_{n}=\left[\begin{array}{cc}
0 & a \\
I_{n-1} & \beta
\end{array}\right]=\left[\begin{array}{cc}
0 & 1 \\
I_{n-1} & 0
\end{array}\right]\left[\begin{array}{cc}
I_{n-1} & 0 \\
0 & a
\end{array}\right]\left[\begin{array}{cc}
I_{n-1} & \beta \\
0 & 1
\end{array}\right]:=P A Q .
$$

Notice that $P$ is a unitary matrix and $Q$ is an invertible matrix. Then $L_{n}^{(1,3)}=(P A Q)^{(1,3)}$ exists if and only if $(A Q)^{(1,3)}$ exists if and only if $A^{(1,3)}$ exists if and only if $a^{(1,3)}$ exists. Indeed, if $(A Q)^{(1,3)}$ exists, then $Q(A Q)^{(1,3)}$ is a $\{1,3\}$-inverse of $A$; conversely, if $A^{(1,3)}$ exists, then $Q^{-1} A^{(1,3)}$ is a $\{1,3\}$-inverse of $A Q$. In this case,

$$
\left[\begin{array}{cc}
-\beta a^{(1,3)} & I_{n-1} \\
a^{(1,3)} & 0
\end{array}\right] \in L_{n}\{1,3\} \text { and } L_{n} L_{n}^{(1,3)}=\left[\begin{array}{cc}
a a^{(1,3)} & 0 \\
0 & I_{n-1}
\end{array}\right] .
$$


From Lemma 2.1, together with, $L_{n}^{\oplus}$ exists if and only if both $L_{n}^{\#}$ and $L_{n}^{(1,3)}$ exist (see [15, Theorem 2.6]), the equivalences of (1)-(3) follow. and

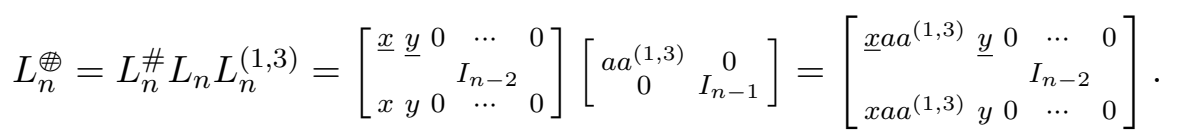

The following result is a characterization for the Moore-Penrose invertibility of a companion matrix, which extends [9, Theorem 3.1] from invertibility to one-sided invertibility. We begin with an auxiliary lemma.

Lemma 2.3. [16] Let $a \in R$ be regular with an inner inverse $a^{-}$. Then the following conditions are equivalent:

(1) $a^{\dagger}$ exists.

(2) $a^{*} a+1-a^{-} a$ is left invertible.

(3) $a^{*} a+1-a^{-} a$ is right invertible.

Theorem 2.4. If $L_{n}=\left[\begin{array}{cc}0 & a \\ I_{n-1} & \beta\end{array}\right]$ with $\beta=\left[a_{1}, a_{2}, \cdots, a_{n-1}\right]^{\mathrm{T}}$ is a companion matrix over $R$. Then the following conditions are equivalent:

(1) $L_{n}^{\dagger}$ exists.

(2) $a$ is regular with an inner inverse $a^{-}, \xi=a^{*} a+1-a^{-} a+\beta^{*} \beta\left(1-a^{-} a\right)$ is left invertible.

(3) $a$ is regular with an inner inverse $a^{-}, \xi=a^{*} a+1-a^{-} a+\beta^{*} \beta\left(1-a^{-} a\right)$ is right invertible.

Proof. Consider $L_{n}=\left[\begin{array}{cc}0 & a \\ I_{n-1} & \beta\end{array}\right]=\left[\begin{array}{cc}a & 0 \\ \beta & I_{n-1}\end{array}\right]\left[\begin{array}{cc}0 & 1 \\ I_{n-1} & 0\end{array}\right]:=A Q$. Since $Q$ is a unitary matrix, then $L_{n}^{\dagger}$ exists if and only if $A^{\dagger}$ exists. From Lemma 2.3, it is known that $A^{\dagger}$ exists if and only if $A$ is regular with an inner inverse $A^{-}$and $A^{*} A+I_{n}-A^{-} A$ is left invertible if and only if $A$ is regular with an inner inverse $A^{-}$and $A^{*} A+I_{n}-A^{-} A$ is right invertible. Observe that $A$ is regular if and only if $a$ is regular. In this case, $\left[\begin{array}{cc}a^{-} & 0 \\ -\beta a^{-} & I_{n-1}\end{array}\right]$ is an inner inverse of $A$ and

$$
A^{*} A+I_{n}-A^{-} A=\left[\begin{array}{cc}
a^{*} a+1-a^{-} a+\beta^{*} \beta & \beta^{*} \\
\beta a^{-} a & I_{n-1}
\end{array}\right]=\left[\begin{array}{cc}
1 & \beta^{*} \\
0 & I_{n-1}
\end{array}\right]\left[\begin{array}{cc}
\xi & 0 \\
0 & I_{n-1}
\end{array}\right]\left[\begin{array}{cc}
1 & 0 \\
\beta a^{-} a & I_{n-1}
\end{array}\right] .
$$

Thus, $A^{\dagger}$ exists if and only if $a$ is regular with an inner inverse $a^{-}$and $\xi$ is left invertible if and only if $a$ is regular with an inner inverse $a^{-}$and $\xi$ is right invertible. We use $\left(A^{*} A+I_{n}-A^{-} A\right)^{-1}$ and $\xi^{-1}$ to denote the inverse of $A^{*} A+I_{n}-A^{-} A$ and $\xi$, respectively. In this case,

$$
\left(A^{*} A+I_{n}-A^{-} A\right)^{-1}=\left[\begin{array}{cc}
1 & 0 \\
-\beta a^{-} a & I_{n-1}
\end{array}\right]\left[\begin{array}{cc}
\xi^{-1} & 0 \\
0 & I_{n-1}
\end{array}\right]\left[\begin{array}{cc}
1 & -\beta^{*} \\
0 & I_{n-1}
\end{array}\right]=\left[\begin{array}{cc}
\xi^{-1} & -\xi^{-1} \beta^{*} \\
-\beta a^{-} a \xi^{-1} & \beta a^{-} a \xi^{-1} \beta^{*}+I_{n-1}
\end{array}\right]
$$

and

$$
A^{\dagger}=\left[A\left(A^{*} A+I_{n}-A^{-} A\right)^{-1}\right]^{*}=\left[\begin{array}{cc}
a \xi^{-1} & -a \xi^{-1} \beta^{*} \\
\beta\left(1-a^{-} a\right) \xi^{-1} & I_{n-1}-\beta\left(1-a^{-} a\right) \xi^{-1} \beta^{*}
\end{array}\right]^{*} .
$$

Hence $L_{n}^{\dagger}=Q^{-1} A^{\dagger}=\left[\begin{array}{cc}-a \xi^{-1} \beta^{*} & a \xi^{-1} \\ I_{n-1}-\beta\left(1-a^{-} a\right) \xi^{-1} \beta^{*} & \beta\left(1-a^{-} a\right) \xi^{-1}\end{array}\right]^{*}$.

From Theorem 2.4 and Lemma 2.1, we can characterize the core invertibility and dual core invertibility of the companion matrix $L_{n}$, since $L_{n}^{\oplus}$ and $L_{n \oplus}$ exist if and only if $L_{n}^{\dagger}$ and $L_{n}^{\#}$ exist [15]. Here we omit the details. 


\section{The pseudo core inverse of a companion matrix}

In this section, our main goal is to characterize the pseudo core invertibility of an $n \times n$ companion matrix over $R$. Let us begin with two auxiliary lemmas.

Lemma 3.1. [4, Theorem 2.12] Let $T \in R^{n \times n}$. Then $T^{(}$exists with ind $(T)=k$ if and only if $k$ is the smallest positive integer such that $T^{k}=X\left(T^{*}\right)^{k+1} T^{k}=Y T^{k+1}$ for some $X, Y \in R^{n \times n}$.

Lemma 3.2. [4, Theorem 2.3] Let $T \in R^{n \times n}$. Then $T^{\circledR}$ exists if and only if $T^{D}$ and $\left(T^{k}\right)^{(1,3)}$ exist, where $k \geq \operatorname{ind}(T)$. In this case, $T^{\complement}=T^{D} T^{k}\left(T^{k}\right)^{(1,3)}$.

Now, let $L_{n}$ be an $n \times n$ companion matrix $\left[\begin{array}{cc}0 & a \\ I_{n-1} & \beta\end{array}\right]$ with $\beta=\left[a_{1}, a_{2}, \cdots, a_{n-1}\right]^{\mathrm{T}}$ and let $T_{k}$ be a $k \times k$ lower triangular Toeplitz matrix $\left[\begin{array}{ccc}a & \\ a_{1} & a & \\ \vdots & \cdots & \\ \vdots & \cdots & a_{1} \\ a_{k-1} & \cdots & a_{1}\end{array}\right]$ over $R$.

Theorem 3.3. The following conditions are equivalent:

(1) $L_{n}^{\circledR}$ exists with ind $\left(L_{n}\right)=k(<n)$.

(2) $k$ is the smallest positive integer such that

$$
\left[\begin{array}{cc}
0 & T_{k} \\
I_{n-k} & 0
\end{array}\right]=X\left[\begin{array}{cc}
0 & I_{n-(k+1)} \\
T_{k+1}^{*} & 0
\end{array}\right]\left[\begin{array}{cc}
0 & T_{k} \\
I_{n-k} & 0
\end{array}\right]=Y\left[\begin{array}{cc}
0 & T_{k+1} \\
I_{n-(k+1)} & W_{[n-(k+1)] \times(k+1)}
\end{array}\right]
$$

for some $X, Y \in R^{n \times n}$, where $W_{[n-(k+1)] \times(k+1)}=\left[\begin{array}{cccc}a_{k+1} & 0 & \cdots & 0 \\ \vdots & & & \\ \vdots & 0 & \cdots & 0\end{array}\right]$.

Proof. Note that

$L_{n}^{k}=\left[\begin{array}{cc}0_{k \times(n-k)} & T_{k} \\ I_{n-k} & 0_{(n-k) \times k}\end{array}\right] Q$ for some invertible matrix $Q$,

$L_{n}^{k+1}=\left[\begin{array}{cc}0_{(k+1) \times[n-(k+1)]} & T_{k+1} \\ I_{n-(k+1)} & 0_{[n-(k+1)] \times(k+1)}\end{array}\right] Q^{\prime}$ for some invertible matrix $Q^{\prime}$

and

$L_{n}^{k+1}=L_{n} L_{n}^{k}=\left[\begin{array}{cc}0 & a \\ I_{n-1} & \beta\end{array}\right]\left[\begin{array}{cc}0_{k \times(n-k)} & T_{k} \\ I_{n-k} & 0_{(n-k) \times k}\end{array}\right] Q=\left[\begin{array}{cc}0_{(k+1) \times[n-(k+1)]} & T_{k+1} \\ I_{n-(k+1)} & W_{[n-(k+1)] \times(k+1)}\end{array}\right] Q$.

Since $Q$ and $Q^{\prime}$ are invertible, then the continued equality

$$
\left[\begin{array}{cc}
0 & T_{k} \\
I_{n-k} & 0
\end{array}\right]=X\left[\begin{array}{cc}
0 & I_{n-(k+1)} \\
T_{k+1}^{*} & 0
\end{array}\right]\left[\begin{array}{cc}
0 & T_{k} \\
I_{n-k} & 0
\end{array}\right]=Y\left[\begin{array}{cc}
0 & T_{k+1} \\
I_{n-(k+1)} & W_{[n-(k+1)] \times(k+1)}
\end{array}\right]
$$

in (2) is equivalent to

$$
L_{n}^{k}=\left[\begin{array}{cc}
0 & T_{k} \\
I_{n-k} & 0
\end{array}\right] Q=X\left[\left(Q^{\prime}\right)^{*}\right]^{-1}\left(Q^{\prime}\right)^{*}\left[\begin{array}{cc}
0 & I_{n-(k+1)} \\
T_{k+1}^{*} & 0
\end{array}\right]\left[\begin{array}{cc}
0 & T_{k} \\
I_{n-k} & 0
\end{array}\right] Q=X\left[\left(Q^{\prime}\right)^{*}\right]^{-1}\left(L_{n}^{k+1}\right)^{*} L_{n}^{k}
$$

and

$$
L_{n}^{k}=\left[\begin{array}{cc}
0 & T_{k} \\
I_{n-k} & 0
\end{array}\right] Q=Y\left[\begin{array}{cc}
0 & T_{k+1} \\
I_{n-(k+1)} & W_{[n-(k+1)] \times(k+1)}
\end{array}\right] Q=Y L_{n}^{k+1} .
$$

According to Lemma 3.1, we derive that (1) is equivalent to (2). 
Theorem 3.4. The following conditions are equivalent:

(1) $L_{n}^{(}$exists with ind $\left(L_{n}\right)=k(\leq n)$.

(2) $k$ is the smallest positive integer such that $T_{k}^{(1,3)}$ exists and $U_{k}=L_{n}^{k}+\left[\begin{array}{cc}I_{k}-T_{k} T_{k}^{(1,3)} & 0 \\ 0 & 0\end{array}\right]$ is invertible.

(3) $k$ is the smallest positive integer such that $T_{k}^{(1,3)}$ exists and $V_{k}=L_{n}^{k}+\left[\begin{array}{ll}0 & 0 \\ 0 & I_{k}-T_{k}^{(1,3)} T_{k}\end{array}\right]$ is invertible.

Proof. Since $L_{n}^{k}=\left[\begin{array}{cc}0_{k \times(n-k)} & T_{k} \\ I_{n-k} & 0_{(n-k) \times k}\end{array}\right] Q$ for some invertible matrix $Q$. Thus, $\left(L_{n}^{k}\right)^{(1,3)}$ exists if and only if $\left[\begin{array}{cc}0 & T_{k} \\ I_{n-k} & 0\end{array}\right]^{(1,3)}$ exists if and only if $T_{k}^{(1,3)}$ exists. In this case, $L_{n}^{k}\left(L_{n}^{k}\right)^{(1,3)}=$ $\left[\begin{array}{cc}T_{k} T_{k}^{(1,3)} & 0 \\ 0 & I_{n-k}\end{array}\right]$ and $\left(L_{n}^{k}\right)^{(1,3)} L_{n}^{k}=\left[\begin{array}{cc}I_{n-k} & 0 \\ 0 & T_{k}^{(1,3)} T_{k}\end{array}\right]$. From [11, Proposition], we know that $L_{n}^{D}$ exists with Drazin index $k(\leq n)$ if and only if $k$ is the smallest positive integer such that $T_{k}$ is regular in $R^{k \times k}$ and $L_{n}^{k}+I_{n}-L_{n}^{k}\left(L_{n}^{k}\right)^{-}$is invertible if and only if $k$ is the smallest positive integer such that $T_{k}$ is regular in $R^{k \times k}$ and $L_{n}^{k}+I_{n}-\left(L_{n}^{k}\right)^{-} L_{n}^{k}$ is invertible. Hence, the equivalences of (1)-(3) follows from Lemma 3.2.

From the above result, we know, before we characterize the pseudo core inverse of a $n \times n$ companion matrix $L_{n}$ in terms of an invertible matrix, we need to get a $\{1,3\}$-inverse of $T_{k}$. In addition, from [6, Theorem 2], we know that $T_{k}^{D}$ exists if and only if $a^{D}$ exists. By Lemma 3.2, if we characterize the pseudo core invertibility (resp. core invertibility) of $T_{k}$, noting that for any positive integer $m, T_{k}^{m}$ is still a lower triangular Toeplitz matrix, then it suffices to characterize a $\{1,3\}$-inverse of a $k \times k$ lower triangular Toeplitz matrix.

In what follows, we specially discuss a $\{1,3\}$-inverse of a $k \times k$ lower triangular Toeplitz matrix.

\section{A $\{1,3\}$-inverse of the lower triangular Toeplitz matrix}

In this section, we discuss a $\{1,3\}$-inverse of a $k \times k$ lower triangular Toeplitz matrix $T_{k}=$ $\left[\begin{array}{cccc}a & & \\ a_{1} & a & \\ \vdots & \ddots & \\ a_{k-1} & \cdots & a_{1} & a\end{array}\right](k \geq 2)$ over $R$. First of all, we should note the following fact.

Example 4.1. Let $T_{2}=\left[\begin{array}{cc}a & 0 \\ a_{1} & a\end{array}\right]$. Then we have the following facts:

(1) $a^{(1,3)}$ exists, which may not imply that $T_{2}^{(1,3)}$ exists. For example, take $T_{2}=\left[\begin{array}{ll}0 & 0 \\ 2 & 0\end{array}\right] \in \mathbb{Z}_{4}^{2 \times 2}$ with transpose as involution. Observe that 0 is $\{1,3\}$-invertible, but $T_{2}$ has no $\{1,3\}$-inverse. (2) $T_{2}^{(1,3)}$ exists, which may not imply that $a^{(1,3)}$ exists. For example, take $T_{2}=\left[\begin{array}{ll}2 & 0 \\ 1\end{array}\right] \in \mathbb{Z}_{4}^{2 \times 2}$ with transpose as involution. Observe that 2 has no $\{1,3\}$-inverse in $\mathbb{Z}_{4}$, but $\left[\begin{array}{ll}2 & 1 \\ 0 & 2\end{array}\right]$ is a $\{1,3\}$ inverse of $T_{2}$.

We recall that $R$ satisfies the Rao condition [14] provided that

$$
a_{1}=\sum_{i=1}^{n} a_{i} a_{i}^{*} \quad \text { implies } a_{i}=0 \quad \text { whenever } i \neq 1 .
$$




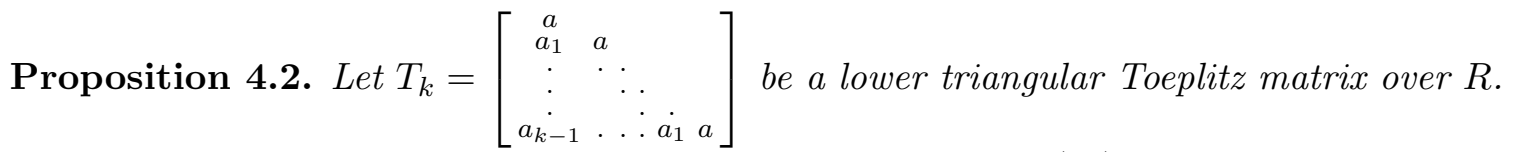

(1) If $a^{(1,3)}$ exists with $a a^{(1,3)} a_{i}=a_{i}, i=1, \cdots, k-1$. Then $T_{k}^{(1,3)}$ exists.

(2) If $R$ satisfies the Rao condition and $T_{k}^{(1,3)}$ exists. Then $a^{(1,3)}$ exists.

Proof. (1) For $k=2, T_{2}=\left[\begin{array}{cc}a & 0 \\ a_{1} & a\end{array}\right]$, if $a a^{(1,3)} a_{1}=a_{1}$, then we can check that

$$
\left[\begin{array}{cc}
a^{(1,3)} & 0 \\
-a^{(1,3)} a_{1} a^{(1,3)} & a^{(1,3)}
\end{array}\right] \text { is a }\{1,3\} \text {-inverse of } T_{2} \text {. }
$$

Suppose that $T_{k-1}^{(1,3)}$ exists. Observe that $T_{k}=\left[\begin{array}{cc}T_{k-1} & 0 \\ \gamma & a\end{array}\right]$, where $\gamma=\left[a_{k-1}, a_{k-2}, \cdots, a_{1}\right]$. From $a a^{(1,3)} a_{i}=a_{i}$, it follows that $\gamma T_{k-1}^{(1,3)}+a X=0$ has a solution $X=-a^{(1,3)} \gamma T_{k-1}^{(1,3)}$. Thus, we can check that $\left[\begin{array}{cc}T_{k-1}^{(1,3)} & 0_{(k-1) \times 1} \\ -a^{(1,3)} \gamma T_{k-1}^{(1,3)} & a^{(1,3)}\end{array}\right]$ is a $\{1,3\}$-inverse of $T_{k}$.

(2) Let $T_{k}=\left[\begin{array}{cc}a & 0 \\ \delta & T_{k-1}\end{array}\right]$, where $\delta=\left[a_{1}, a_{2}, \cdots, a_{k-1}\right]^{\mathrm{T}}$ and suppose $\left[\begin{array}{cc}x_{1} & x_{2} \\ x_{3} & x_{4}\end{array}\right] \in T_{k}\{1,3\}$. Since $R$ satisfies the Rao condition and $T_{k}^{(1,3)}$ exists, by [14, Lemma 1], $T_{k} T_{k}^{(1,3)}=\operatorname{diag}\left(e_{1}, \cdots, e_{k}\right)$, $e_{i}^{2}=e_{i}=e_{i}^{*}$. Checking equations $\left(T_{k} T_{k}^{(1,3)}\right)^{*}=T_{k} T_{k}^{(1,3)}$ and $T_{k} T_{k}^{(1,3)} T_{k}=T_{k}$, we have $\left(a x_{1}\right)^{*}=a x_{1}$ and $a x_{1} a=a$. Hence $f_{1}^{\mathrm{T}}\left(T_{k}\right)^{(1,3)} f_{1} \in a\{1,3\}$, where $f_{1}=[1,0, \cdots, 0]^{\mathrm{T}}$.

In the following, we give characterizations for $T_{k}$ to be $\{1,3\}$-invertible, under some prescribed conditions.

Theorem 4.3. Let $T_{k}=\left[\begin{array}{ccc}a & & \\ a_{1} & a & \\ \vdots & \cdots & \\ a_{k-1} & \ldots & a_{1}\end{array}\right]$ be a lower triangular Toeplitz matrix over $R$ such that $a^{(1,3)}$ and $T_{k-1}^{(1,3)}$ exist.

(1) Suppose $w=\left(I_{k-1}-T_{k-1} T_{k-1}^{(1,3)}\right) \delta\left(1-a^{(1,2,3)} a\right)$ such that $w^{(1,3)}$ exists, where $\delta=\left[a_{1}, a_{2}, \cdots, a_{k-1}\right]^{\mathrm{T}}$. Then $T_{k}^{(1,3)}$ exists if and only if

$$
\alpha=1+\left(\delta a^{(1,2,3)}\right)^{*}\left(I_{k-1}-T_{k-1} T_{k-1}^{(1,3)}-w w^{(1,3)}\right) \delta a^{(1,2,3)} \text { is invertible. }
$$

(2) Suppose $s=\left(1-a a^{(1,3)}\right) \gamma\left(I_{k-1}-T_{k-1}^{(1,2,3)} T_{k-1}\right)$ such that ${ }^{(1,3)}$ exists, where $\gamma=\left[a_{k-1}, a_{k-2}, \cdots, a_{1}\right]$. Then $T_{k}^{(1,3)}$ exists if and only if

$$
\beta=I_{k-1}+\left(\gamma T_{k-1}^{(1,2,3)}\right)^{*}\left(1-a a^{(1,3)}-s s^{(1,3)}\right) \gamma T_{k-1}^{(1,2,3)} \text { is invertible. }
$$

Proof. (1) Since $a^{(1,3)}, T_{k-1}^{(1,3)}, w^{(1,3)}$ exist, then $a^{(1,2,3)}, T_{k-1}^{(1,2,3)}, w^{(1,2,3)}$ exist and $a a^{(1,3)}=$ $a a^{(1,2,3)}, T T_{k-1}^{(1,3)}=T T_{k-1}^{(1,2,3)}, w w^{(1,3)}=w w^{(1,2,3)}$. Let

$$
T_{k}=\left[\begin{array}{ll}
a & 0 \\
\delta & T_{k-1}
\end{array}\right]=\left[\begin{array}{cc}
1 & 0 \\
{\left[I_{k-1}-T_{k-1} T_{k-1}^{(1,3)}\right] \delta a^{(1,2,3)}} & I_{k-1}
\end{array}\right]\left[\begin{array}{cc}
a & 0 \\
w & T_{k-1}
\end{array}\right]\left[\begin{array}{cc}
1 & 0 \\
T_{k-1}^{(1,2,3)} \delta & I_{k-1}
\end{array}\right]:=P A Q .
$$


It is easy to check that $\left[\begin{array}{cc}a^{(1,2,3)} & {\left[1-a^{(1,2,3)} a\right] w^{(1,2,3)}} \\ 0 & T_{k-1}^{(1,2,3)}\end{array}\right]$ is a $\{1,3\}$-inverse of $A$. By computation,

$$
P\left(I-A A^{(1,3)}\right)=\left[\begin{array}{cc}
1-a a^{(1,3)} & 0 \\
0 & I_{k-1}-T_{k-1} T_{k-1}^{(1,3)}-w w^{(1,3)}
\end{array}\right]=I-A A^{(1,3)} .
$$

According to [2, Theorem 3.1], $P A^{(1,3)}$ exists if and only if

$$
\begin{aligned}
U & =P^{*} P A A^{(1,3)}+I-A A^{(1,3)} \\
& =\left[\begin{array}{cc}
1+\left(\delta a^{(1,2,3)}\right)^{*}\left[I_{k-1}-T_{k-1} T_{k-1}^{(1,3)}\right] \delta a^{(1,2,3)} & \left(\delta a^{(1,2,3)}\right)^{*} w w^{(1,3)} \\
{\left[I_{k-1}-T_{k-1} T_{k-1}^{(1,3)}\right] \delta a^{(1,2,3)}} & I_{k-1}
\end{array}\right] \\
& =\left[\begin{array}{ccc}
1 & \left(\delta a^{(1,2,3)}\right)^{*} w w^{(1,3)} \\
0 & I_{k-1}
\end{array}\right]\left[\begin{array}{cc}
\alpha & 0 \\
0 & I_{k-1}
\end{array}\right]\left[\begin{array}{cc}
1 & 0 \\
{\left[I_{k-1}-T_{k-1} T_{k-1}^{(1,3)}\right] \delta a^{(1,2,3)}} & I_{k-1}
\end{array}\right]
\end{aligned}
$$

is invertible if and only if $\alpha$ is invertible. In this case, $A^{(1,3)} U^{-1} P^{*}$ is a $\{1,3\}$-inverse of $P A$, and then $Q^{-1} A^{(1,3)} U^{-1} P^{*}=\left[\begin{array}{ll}x_{1} & x_{2} \\ x_{3} & x_{4}\end{array}\right]$ is a $\{1,3\}$-inverse of $T_{k}$, where

$$
\begin{aligned}
x_{1}= & a^{(1,2,3)} \alpha^{-1}-\left[1-a^{(1,2,3)} a\right] w^{(1,2,3)} \delta a^{(1,2,3)} \alpha^{-1} ; \\
x_{2}= & {\left[1-a^{(1,2,3)} a\right] w^{(1,2,3)}+\left[1-w^{(1,2,3)} \delta+a^{(1,2,3)} a w^{(1,2,3)} \delta\right] } \\
& \times a^{(1,2,3)} \alpha^{-1}\left(\delta a^{(1,2,3)}\right)^{*}\left[I_{k-1}-T_{k-1} T_{k-1}^{(1,3)}-w w^{(1,3)}\right] ; \\
x_{3}= & {\left[T_{k-1}^{(1,2,3)} \delta\left(1-a^{(1,2,3)} a\right) w^{(1,2,3)}-T_{k-1}^{(1,2,3)}\right] \delta a^{(1,2,3)} \alpha^{-1} ; } \\
x_{4}= & T_{k-1}^{(1,2,3)}-T_{k-1}^{(1,2,3)} \delta\left[1-a^{(1,2,3)} a\right] w^{(1,2,3)}+T_{k-1}^{(1,2,3)}\left[\delta\left(1-a^{(1,2,3)} a\right) w^{(1,2,3)}-I_{k-1}\right] \\
& \times \delta a^{(1,2,3)} \alpha^{-1}\left(\delta a^{(1,2,3)}\right)^{*}\left[I_{k-1}-T_{k-1} T_{k-1}^{(1,3)}-w w^{(1,3)}\right] .
\end{aligned}
$$

(2) It is analogous.

Corollary 4.4. Let $T_{2}=\left[\begin{array}{cc}a & 0 \\ a_{1} & a\end{array}\right]$ such that $a^{(1,3)}$ exists. Suppose $w=\left[1-a a^{(1,3)}\right] a_{1}[1-$ $\left.a^{(1,2,3)} a\right]$ such that $w^{(1,3)}$ exists. Then $T_{2}\{1,3\} \neq \emptyset$ if and only if $\alpha=1+\left[a_{1} a^{(1,2,3)}\right]^{*}[1-$ $\left.a a^{(1,3)}-w w^{(1,3)}\right] a_{1} a^{(1,2,3)}$ is invertible.

Corollary 4.5. Let $T_{3}=\left[\begin{array}{ccc}a & 0 & 0 \\ a_{1} & a & 0 \\ a_{2} & a_{1} & a\end{array}\right]$ such that $a^{(1,3)}$ and $T_{2}^{(1,3)}$ exist.

(1) Suppose $w=\left(I_{2}-T_{2} T_{2}^{(1,3)}\right)\left[\begin{array}{l}a_{1} \\ a_{2}\end{array}\right]\left(1-a^{(1,2,3)} a\right)$ such that $w^{(1,3)}$ exists. Then $T_{3}^{(1,3)}$ exists if and only if $\alpha=1+\left(\left[\begin{array}{c}a_{1} \\ a_{2}\end{array}\right] a^{(1,2,3)}\right)^{*}\left(I_{2}-T_{2} T_{2}^{(1,3)}-w w^{(1,3)}\right)\left[\begin{array}{c}a_{1} \\ a_{2}\end{array}\right] a^{(1,2,3)}$ is invertible.

(2) Suppose $s=\left(1-a a^{(1,3)}\right)\left[a_{2} a_{1}\right]\left(I_{2}-T_{2}^{(1,2,3)} T_{2}\right)$ such that $s^{(1,3)}$ exists. Then $T_{3}^{(1,3)}$ exists if and only if $\beta=I_{2}+\left(\left[\begin{array}{ll}a_{2} & a_{1}\end{array}\right] T_{2}^{(1,2,3)}\right)^{*}\left(1-a a^{(1,3)}-s s^{(1,3)}\right)\left[\begin{array}{ll}a_{2} & a_{1}\end{array}\right] T_{2}^{(1,2,3)}$ is invertible.

$R$ satisfies the extended Rao condition $[5,14]$ provided that

$$
1=\sum a_{i} a_{i}^{*} \quad \text { implies } a_{i}=0 \quad \text { for every index } i \text { except possibly one. }
$$


Theorem 4.6. Let $T_{k}=\left[\begin{array}{ccc}a & & \\ a_{1} & a & \\ \vdots & \cdots & \ddots \\ a_{k-1} & \ldots & a_{1} \\ \end{array}\right] \neq 0$ be a non-right invertible lower triangular Toeplitz matrix over $R$. If $R$ satisfies the extended Rao condition. Then the following conditions are equivalent:

(1) $T_{k}^{(1,3)}$ exists.

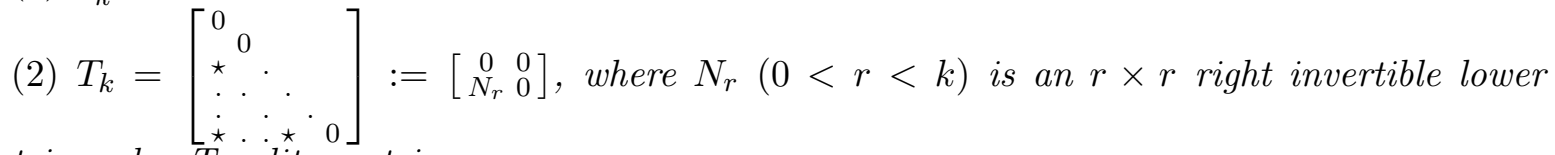
triangular Toeplitz matrix.

Proof. $\quad(1) \Rightarrow(2)$ If $T_{k}^{(1,3)}$ exists, then $T_{k}^{(1,2,3)}$ exists. By [14, Lemma 2],

$$
T_{k} T_{k}^{(1,2,3)}=\operatorname{diag}\left(e_{1}, \cdots, e_{k}\right), \text { where } e_{i}=0 \text { or } 1 .
$$

Since $T_{k}$ is non-right invertible, there is a permutation matrix $P$ such that

$$
P^{-1} T_{k} T_{k}^{(1,2,3)} P=\left[\begin{array}{ll}
0 & 0 \\
0 & I_{r}
\end{array}\right] .
$$

Observe that

$$
P^{-1} T_{k}=P^{-1} T_{k} T_{k}^{(1,2,3)} P P^{-1} T_{k}=\left[\begin{array}{ll}
0 & 0 \\
0 & I_{r}
\end{array}\right] P^{-1} T_{k}:=\left[\begin{array}{c}
0 \\
B_{r \times k}
\end{array}\right]
$$

and

$$
T_{k}^{(1,2,3)} P=T_{k}^{(1,2,3)} P P^{-1} T_{k} T_{k}^{(1,2,3)} P=T_{k}^{(1,2,3)} P\left[\begin{array}{ll}
0 & 0 \\
0 & I_{r}
\end{array}\right]:=\left[\begin{array}{ll}
0 & C_{k \times r}
\end{array}\right] .
$$

From (*), it follows that $B_{r \times k} C_{k \times r}=I_{r}$. Hence $T_{k}=P\left[\begin{array}{c}0 \\ B_{r \times k}\end{array}\right]$, where $B_{r \times k}$ is right invertible.

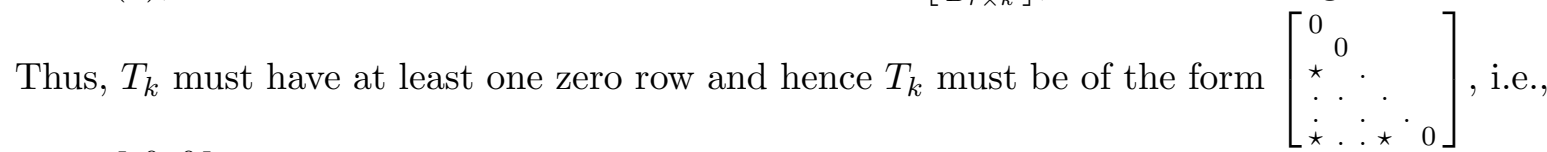
$T_{k}=\left[\begin{array}{cc}0 & 0 \\ N_{r} & 0\end{array}\right]$, where $N_{r}$ is an $r \times r$ lower triangular Toeplitz matrix and the first row of $N_{r}$ is not zero, which mean that each row of $N_{r}$ is not zero. Suppose $T_{k}^{(1,2,3)}=\left[\begin{array}{ll}L_{1} & L_{2} \\ L_{3} & L_{4}\end{array}\right]$, then $T_{k} T_{k}^{(1,2,3)}=\left[\begin{array}{cc}0 & 0 \\ N_{r} L_{1} & N_{r} L_{2}\end{array}\right]$. Again by [14, Lemma 2], $T_{k} T_{k}^{(1,2,3)}=\left[\begin{array}{cc}0 & 0 \\ 0 & N_{r} L_{2}\end{array}\right]:=\left[\begin{array}{ll}0 & 0 \\ 0 & H_{r}\end{array}\right]$, where $H_{r}=\operatorname{diag}\left(e_{1}, \cdots, e_{r}\right), e_{i}=0$ or 1 . From $T_{k} T_{k}^{(1,2,3)} T_{k}=\left[\begin{array}{cc}0 & 0 \\ 0 & H_{r}\end{array}\right]\left[\begin{array}{cc}0 & 0 \\ N_{r} & 0\end{array}\right]=\left[\begin{array}{cc}0 & 0 \\ N_{r} & 0\end{array}\right]=T_{k}$, it follows that $H_{r} N_{r}=N_{r}$. Since none of the rows of $N_{r}$ is zero, then $H_{r}=I_{r}$. Therefore $N_{r}$ is right invertible.

$(2) \Rightarrow(1)$ If $T_{k}=\left[\begin{array}{cc}0 & 0 \\ N_{r} & 0\end{array}\right]$, then $\left[\begin{array}{cc}0 & S_{r} \\ 0 & 0\end{array}\right]$ is a $\{1,3\}$-inverse of $T_{k}$, where $S_{r}$ stands for the right inverse of $N_{r}$.

\section{The commutative case}

In this section, the matrices considered are over a commutative $*$-ring $R$. 
Theorem 5.1. Let $T_{k}=\left[\begin{array}{ccc}a & & \\ a_{1} & a & \\ \vdots & \cdots & \\ \vdots & \ddots & \\ a_{k-1} & \ldots & a_{1}\end{array}\right]$ be a lower triangular Toeplitz matrix over $R$. If a and $a_{i}(i=1, \cdots, k-1)$ are $\{1,3\}$-invertible in $R$. Then $T_{k}$ is $\{1,3\}$-invertible in $R^{k \times k}$.

Proof. Observe that if $e^{2}=e=e^{*}$ in $R$ and $X_{1}, X_{2}$ are $\{1,3\}$-invertible in $R^{k \times k}$, then $X_{1} e+X_{2}(1-e)$ is $\{1,3\}$-invertible in $R^{k \times k}$ and $X_{1}^{(1,3)} e+X_{2}^{(1,3)}(1-e)$ are all $\{1,3\}$-inverses of $X_{1} e+X_{2}(1-e)$. Conversely, if $X_{1} e+X_{2}(1-e)$ is $\{1,3\}$-invertible in $R^{k \times k}$, then both $X_{1} e$ and $X_{2}(1-e)$ are $\{1,3\}$-invertible in $R^{k \times k}$.

Since all the components of $T_{k}$ are $\{1,3\}$-invertible in $R$, then they must be $\{1,2,3\}$ invertible. Hence we factorize $T_{k}$ as

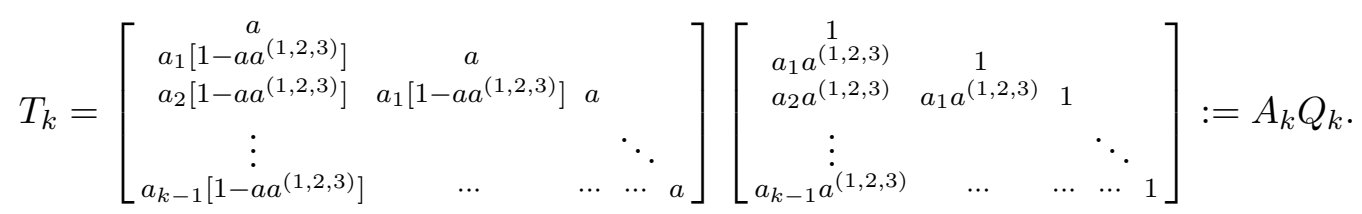

Note that $Q_{k}$ is invertible, then $T_{k}^{(1,3)}$ exists if and only if $A_{k}^{(1,3)}$ exists. In this case, $Q_{k}^{-1} A_{k}^{(1,3)}$ is a $\{1,3\}$-inverse of $T_{k}$. Consider the factorization of $A_{k}$ :

$$
A_{k}=\left[\begin{array}{ccc}
a & & \\
& \ddots & \\
& & a
\end{array}\right] a a^{(1,2,3)}+\left[\begin{array}{cccc:c}
0 & \cdots & \cdots & 0 & 0 \\
\hdashline a_{1} & & & 0 \\
a_{2} & a_{1} & & \vdots \\
\vdots & & \ddots & \vdots \\
a_{k-1} & \cdots & \cdots & a_{1} & 0
\end{array}\right]\left[1-a a^{(1,2,3)}\right] .
$$

Thus, we derive a reduction formula of a $\{1,3\}$-inverse of $T_{k}$.

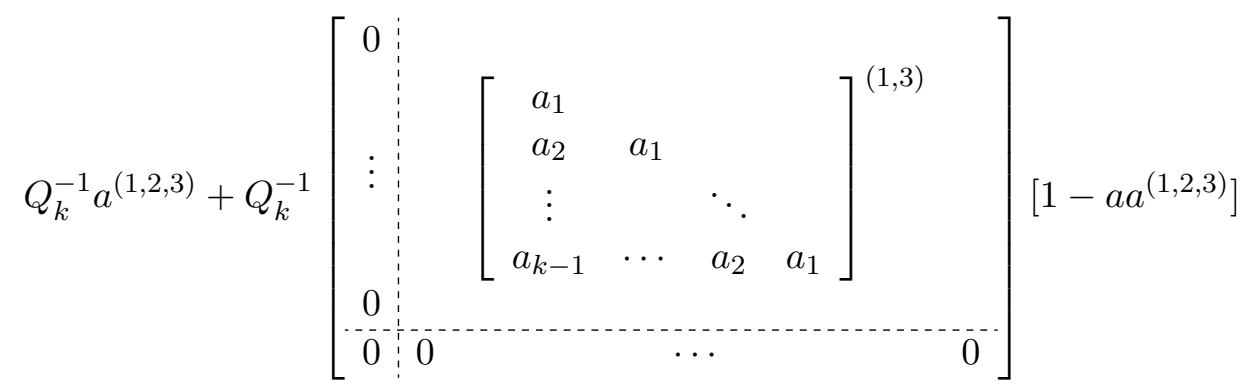

$$
\begin{aligned}
& =Q_{k}^{-1} a^{(1,2,3)}+Q_{k}^{-1}\left[1-a a^{(1,2,3)}\right] \times
\end{aligned}
$$




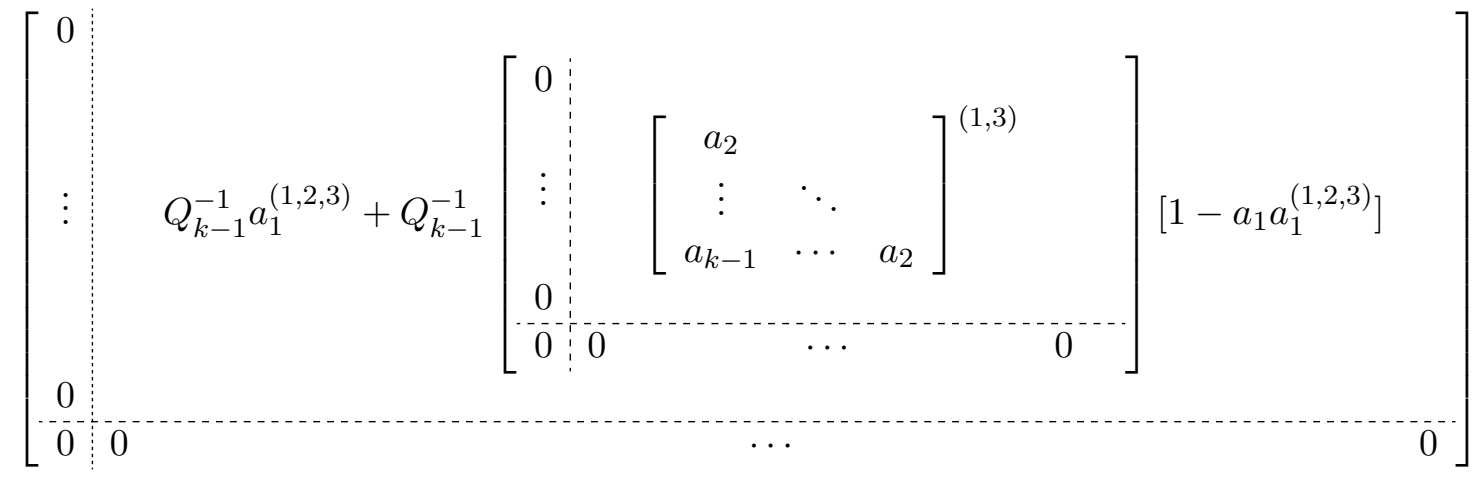

is a $\{1,3\}$-inverse of $T_{k}$.

Corollary 5.2. Under the conditions of Theorem 5.1.

(1) $\left[\begin{array}{cc}a & 0 \\ a_{1} & a\end{array}\right]^{(1,3)}=\left[\begin{array}{cc}a^{(1,2,3)} & a_{1}^{(1,2,3)}\left[1-a a^{(1,2,3)}\right] \\ -a_{1}\left[a^{(1,2,3)}\right]^{2} & a^{(1,2,3)}\end{array}\right]$,

(2) $\left[\begin{array}{ccc}a & 0 & 0 \\ a_{1} & a & 0 \\ a_{2} & a_{1} & a\end{array}\right]^{(1,3)}=\left[\begin{array}{ccc}a^{(1,2,3)} & a_{1}^{(1,2,3)}\left[1-a a^{(1,2,3)}\right] & a_{2}^{(1,2,3)}\left[1-a_{1} a_{1}^{(1,2,3)}\right]\left[1-a a^{(1,2,3)}\right] \\ -a_{1}\left[a^{(1,2,3)}\right]^{2} & a^{(1,2,3)} & a_{1}^{(1,2,3)}\left[1-a a^{(1,2,3)}\right] \\ a_{1}^{2}\left(a^{(1,2,3)}\right)^{3}-a_{2}\left(a^{(1,2,3)}\right)^{2} & -a_{1}\left[a^{(1,2,3)}\right]^{2} & a^{(1,2,3)}\end{array}\right]$ $+\left[\begin{array}{ccc}0 & 0 & 0 \\ 0 & -a_{2}\left[a_{1}^{(1,2,3)}\right]^{2}\left[1-a a^{(1,2,3)}\right] & 0 \\ 0 & 0 & 0\end{array}\right]$,

(3) $\left[\begin{array}{cccc}a_{1} & 0 & 0 & 0 \\ a_{1} & a & 0 & 0 \\ a_{2} & a_{1} & a & 0 \\ a_{3} & a_{2} & a_{1} & a\end{array}\right]^{(1,3)}$

$$
\begin{aligned}
& =\left[\begin{array}{cccc}
a^{(1,2,3)} & a_{1}^{(1,2,3)} e & a_{2}^{(1,2,3)} e_{1} e & a_{3}^{(1,2,3)} e_{2} e_{1} e \\
-a_{1}\left[a^{(1,2,3)}\right]^{2} & a^{(1,2,3)} & a_{1}^{(1,2,3)} e & a_{2}^{(1,2,3)} e_{1} e \\
a_{1}^{2}\left(a^{(1,2,3)}\right)^{3}-a_{2}\left(a^{(1,2,3)}\right)^{2} & -a_{1}\left[a^{(1,2,3)}\right]^{2} & a^{(1,2,3)} & a^{(1,2,3)} e \\
2 a_{1} a_{2}\left[a^{(1,2,3)}\right]^{3}-a_{3}\left[a^{(1,2,3)}\right]^{2}-a_{1}^{3}\left[a^{(1,2,3)}\right]^{4} & a_{1}^{2}\left(a^{(1,2,3)}\right)^{3}-a_{2}\left(a^{(1,2,3)}\right)^{2} & -a_{1}\left[a^{(1,2,3)}\right]^{2} & a^{(1,2,3)}
\end{array}\right] \\
& +\left[\begin{array}{lccc}
0 & 0 & 0 & 0 \\
0 & -a_{2}\left[a_{1}^{(1,2,3)}\right]^{2} e & -a_{3}\left[a_{2}^{(1,2,3)}\right]^{2} e_{1} & 0 \\
0\left[a_{2}^{2}\left[a_{1}^{(1,2,3)}\right]^{3}-a_{3}\left[a_{1}^{(1,2,3)}\right]^{2}\right] e-a_{2}\left[a^{(1,2,3)}\right]^{2} & -a_{2}\left[a_{1}^{(1,2,3)}\right]^{2} e & 0 \\
0 & 0 & 0 & 0
\end{array}\right],
\end{aligned}
$$

where $a=a_{0}, e=e_{0}, e_{i}=1-a_{i} a_{i}^{(1,2,3)}, i=0,1,2$.

Remark that in the above three cases, the special $\{1,3\}$-inverse $T_{k}^{(1,3)}$ of $T_{k}$ is the sum of a Toeplitz matrix and a zero-bordered Toeplitz matrix.

\section{Acknowledgements}

This research is supported by the National Natural Science Foundation of China (No.11771076, No.11471086), the Scientific Innovation Research of College Graduates in Jiangsu Province (No.KYZZ16_0112). 


\section{References}

[1] O.M. Baksalary, G. Trenkler. Core inverse of matrices, Linear Multilinear Algebra, 58 (6) (2010) 681-697.

[2] N. Castro-Gonzalez, J.L. Chen, L. Wang. Further results on generalized inverses in rings with involution, Electron. J. Linear Algebra, 30 (2015) 118-134.

[3] J.L. Chen, H.H. Zhu, P. Patrício, Y.L. Zhang. Characterizations and representations of core and dual core inverses, Canad. Math. Bull., 60 (2) (2017) 269-282.

[4] Y.F. Gao, J.L. Chen. Pseudo core inverses in rings with involution, Comm. Algebra, 46 (1) (2018) 38-50.

[5] M.C. Gouveia. Generalized invertibility of Hankel and Toeplitz matrices, Linear Algebra Appl., 193 (1993) 95-106.

[6] R.E. Hartwig, J. Shoaf. Group inverses and Drazin inverses of bidiagonal and triangular Toeplitz matrices, J. Austral. Math. Soc., 24 (Series A) (1977) 10-34.

[7] T.T. Li, J.L. Chen. Characterizations of core and dual core inverses in rings with involution, Linear Multilinear Algebra. DOI: 10.1080/03081087.2017.1320963.

[8] K. Manjunatha Prasad, K.S. Mohana. Core-EP inverse, Linear Multilinear Algebra, 62 (2014) $792-802$.

[9] P. Patrício. The Moore-Penrose inverse of a companion matrix, Linear Algebra Appl., 437 (2012) 870-877.

[10] P. Patrício, R. Puystjens. About the von Neumann regularity of triangular block matrices, Linear Algebra Appl., 332-334 (2001) 485-502.

[11] R. Puystjens, M.C. Gouveia. Drazin invertibility for matrices over an arbitrary ring, Linear Algebra Appl., 385 (2004) 105-116.

[12] R. Puystjens, R.E. Hartwig. The group invers of a companion matrix, Linear Multilinear Algebra, 43 (1997) 137-150.

[13] D.S. Rakić, N.Č. Dinčić, D.S. Djordjević. Group, Moore-Penrose, core and dual core inverse in rings with involution, Linear Algebra Appl., 463 (2014) 115-133.

[14] D.W. Robinson, R. Puystjens, J. Van Geel. Categories of matrices with only obvious Moore-Penrose inverses, Linear Algebra Appl., 97 (1987) 93-102.

[15] S.Z. Xu, J.L. Chen, X.X. Zhang. New characterizations for core inverses in rings with involution, Front. Math. China, 12 (1) (2017) 231-246.

[16] H.H. Zhu, J.L. Chen, P. Patrício. Further results on the inverse along an element in semigroups and rings, Linear and Multilinear Algebra, 64 (2016) 393-403. 